\title{
The Asymptotic Behavior of a Variation of Polarized Hodge Structure
}

\author{
By \\ Masaki KASHIWARA*
}

\section{Introduction}

0.1. The purpose of this paper is to give the asymptotic behavior of variation of polarized Hodge structures in the several-dimensional case. We do not discuss here why and how the notion of variation of Hodge structures arises and it is developed by P. A. Griffiths, P. Deligne, W. Schmid and others. What motivates us is to generalize Zucker's result to the several-dimensional case. His result is as follows: the cohomology groups of a variation of Hodge structure on the compact curve with finite singular points have also a Hodge structure. He proceeds his proof as follows. As an analytic tool, he uses the harmonic analysis (Hodge-Kodaira theory) and as a geometric tool he uses W. Schmid's result that we discuss later. By using the Kähler metric on the curve which behaves with the special property at singular points and the Hermitian metric of the vector bundle which arises from the polarization of Hodge structure, he succeeds to express the cohomology groups of the variation as the $L^{2}$-cohomology groups. Since the $L^{2}$-cohomology group is isomorphic to the space of harmonic forms, by decomposing harmonic forms into $(p, q)$-forms, he obtains the Hodge decomposition of the cohomology group of Hodge structure. However, in order to prove the first step-to express the cohomology group by $L^{2}$-cohomology group-he is obliged to use the result of W. Schmid on the asymptotic behavior of variations of Hodge structures at singularity.

In this paper, we generalize W. Schmid's result to the several-

Received April 19, 1985.

* Research Insitute for Mathematical Sciences, Kyoto University, Kyoto 606, Japan. 
dimensional case and in the forthcoming paper, we discuss the generalization of Zucker's result.

0.2. Now we are going to recall Schmid's result. For an integer n, a Hodge structure of weight $n$ is a couple $\left(H_{Z}, F\right)$ consisting of a finitely generated $\boldsymbol{Z}$-module $H_{Z}$ and a finite decreasing filtration $F$ of $H_{C}=\boldsymbol{C} \underset{Z}{\otimes} H_{Z}$ such that $H_{C}=\bigoplus_{n=p+q}\left(F^{p} \cap \bar{F}^{q}\right)$. Here, $\bar{F}$ is the complex conjugate of $F$. The Weil operator $\mathrm{G}$ is the automorphism given by $\left.\mathrm{C}\right|_{H^{p q}}=i^{p-q}$ where $H^{p, q}=F^{b} \cap \vec{F}^{q}$. A polarization $S$ is a non-degenerate bilinear form on $H_{Q}=Q \bigotimes_{Z} H_{Z}$ such that $S\left(F^{p}, F^{n+1-p}\right)=0$ and that $S(\mathrm{C} u, \bar{v})$ is a positive definite Hermitian form on $H_{c}$.

Let $X$ be a complex manifold. A variation of Hodge structure of weight $n$ on $X$ consists of data $\left(H_{Z}, F, S\right): H_{Z}$ is a local system on $X$ and $S$ is a non-degenerate bilinear form $S: H_{Q} \otimes H_{Q} \rightarrow \boldsymbol{Q}_{X}$ and $F$ is a finite filtration of holomorphic vector bundles such that at any point $x$ the stalk $\left(H_{Z}, F, S\right)$ gives a polarized Hodge structure and, for any holomorphic vector field $v, v F^{p} \subset F^{p-1}$.

Let $D$ be the open unit disc of $\boldsymbol{C}$ and $D^{*}$ the pictured disc. Let $\left(H_{Z}, F, S\right)$ be a variation of Hodge structure and let $M$ be the monodromy of $H_{Q}$. Then $M$ is quasi-unipotent (i. e. its eigenvalues are the root of unity).

Set $N=\frac{1}{m} \log M^{m}$ taking $m \geq 1$ so that $M^{m}$ is unipotent. Then $N$ is a nilpotent endomorphism of $H_{Q}$. Let $W(N)$ be the monodromy weight filtration, i. e. the unique filtration such that $N W_{k}(N) \subset W_{k-2}(N)$ and $N^{k}: g r_{k}^{W(N)} \cong g r_{-k}^{W(N)}$.

Then the theorem of W. Schmid says

Theorem. For a flat section $u \in W_{k}$ with $u \notin W_{k-1}$, we have

$$
|u|_{z}^{2} \sim(-\log |z|)^{k} \text { when } z \rightarrow 0 .
$$

Here ||$_{\boldsymbol{z}}$ is the norm given by the polarization of the Hodge structure at $z \in D^{*}$.

In this paper, we give its generalization to several-dimensional case. To simplify the explanation, we consider the two-dimensional case. Let us consider a variation of Hodge structure on $\left(z_{1}, z_{2}\right) \in D^{*} \times D^{*}$. Let $M_{j}(j=1,2)$ be the monodromy at $z_{j}=0$ and define $N_{j}$ from $M_{j}$ 
just as $N$ and $M$ in the one-dimensional case. Let $W_{0}, W_{1}$ and $W_{2}$ be the monodromy weight filtrations of $N_{1}+N_{2}, N_{1}$ and $N_{2}$, respectively。 We divide $D^{*} \times D^{*}$ into two parts

$$
A_{1}=\left\{z \in D^{*} \times D^{*} ; \log \left|z_{1}\right| / \log \left|z_{2}\right|>\varepsilon\right\}
$$

and

$$
A_{2}=\left\{z \in D^{*} \times D^{*} ; \log \left|z_{2}\right| / \log \left|z_{1}\right|>\varepsilon\right\}
$$

Theorem. Let us decompose $H_{c}=\oplus U_{p, q}$ with $W_{0 k}=\bigoplus_{p \leqq k} U_{p, q}$ and $W_{1 k}=$ $\oplus U_{p, q}$ and take a metric | | on $H_{c}$. Then for a flat section $u=\sum u_{p, q}$ of $H_{c}$ with $u_{p, q} \in U_{p, q}$, we have

$$
|u|_{z}^{2} \sim \sum_{p, q}\left(-\log \left|z_{2}\right|\right)^{p-q}\left(-\log \left|z_{1}\right|\right)^{q}\left|u_{p q}\right|^{2} \text { for } z \in A_{1} \text { 。 }
$$

The estimate on $A_{2}$ is similar. For the more precise statement see Theorem 2.4.2, 3.4.1 and 3.4.2.

The author would like to express his gratitude to Professor $T$. Kawai for helpful discussions.

After he finished this paper, the author received a preprint by E. Cattani, A. Kaplan and W. Schmid which contains the same result. The proofs are different.

\section{§1. Filtrations}

1. 1. Let $A$ be an abelian category. A finite (decreasing) filtration $F$ of an object $M$ of $\boldsymbol{A}$ is by definition a decreasing sequence $\left\{F^{p}\right\}$ of subobjects of $M$ such that $F^{p}=M$ for $p \ll 0$ and $F^{p}=0$ for $p \gg 0$. If there is no fear of confusion, we omit the phrase "finite". We write $g r_{F}^{p}=F^{p} / F^{p+1}$. As usual, by $F_{p}=F^{-p}$, we interchange freely increasing filtrations and decreasing filtrations.

1.2. Let $T$ be an exact contravariant functor from $A$ to another abelian category. For a filtration $F$ of an object $M$ of $A$, let us denote by $T(F)$ the filtration of $T(M)$ given by

$$
T(F)^{p}=T\left(M / F^{1-p}\right),
$$

so that we have

$$
g r_{T(F)}^{p}=T\left(g r_{F}^{-p}\right) .
$$

1.3. Let $F_{1}$ and $F_{2}$ be two filtrations. 
Lemma 1.3.1. For any $k$ we have

$$
\sum_{k=p+q} F_{1}^{p} \cap F_{2}^{q}=\bigcap_{p+q=k+1}\left(F_{1}^{p}+F_{2}^{q}\right)
$$

Proof. Set $V^{k}$ (resp. $V^{\prime k}$ ) the left-hand side (resp. right-hand side). We have $V^{k} \subset V^{\prime k}$. In fact, it is enough to show $F_{1}^{b} \cap F_{2}^{q} \subset F_{1}^{\phi^{\prime}}+F_{2}^{q^{\prime}}$ for $k=p+q$ and $k+1=p^{\prime}+q^{\prime}$. If $p \geqq p^{\prime}$ or $q \geqq q^{\prime}$, this is true. Hence we may assume $p \leqq p^{\prime}-1$ and $q \leqq q^{\prime}-1$. Then $k=p+q \leqq\left(p^{\prime}-1\right)+\left(q^{\prime}-1\right)$ $=k-1$, which is a contradiction.

Now we shall show $V^{\prime k} \subset V^{k}$. In order to see this, it is enough to prove

$$
V^{\prime k} \cap F_{2}^{q} \subset V^{k}+F_{2}^{q+1}
$$

Now, we have

$$
V^{\prime k} \cap F_{2}^{q} \subset\left(F_{1}^{k-q}+F_{2}^{q+1}\right) \cap F_{2}^{q} \subset F_{1}^{k-q} \cap F_{2}^{q}+F_{2}^{q+1} \subset V^{k}+F_{2}^{q+1} .
$$

Q. E. D.

Definition 1.3.1. (Steenbrink-Zucker $[\mathrm{S}-\mathrm{Z}]$ ). We define the amalgum $F_{1} * F_{2}$ of $F_{1}$ and $F_{2}$ by

$$
\left(F_{1} * F_{2}\right)^{k}=\sum_{k=p+q} F_{1}^{p} \cap F_{2}^{q}=\sum_{p+q=k+1}\left(F_{1}^{p}+F_{2}^{q}\right) .
$$

Remark 1.3.2. (i) This notion is self-dual, i. e. for an exact contravariant functor $T, T\left(F_{1} * F_{2}\right)=T\left(F_{1}\right) * T\left(F_{2}\right)$.

(ii) For three filtrations $F_{1}, F_{2}$ and $F_{3}$, the relation, $\left(F_{1} * F_{2}\right) * F_{3}=$ $F_{1} *\left(F_{2} * F_{3}\right)$ does not hold in general. Its sufficient condition is discussed in $\S 1.6$.

1.4. Two filtrations $F_{1}$ and $F_{2}$ of $M$ are called $n$-opposed (see Deligne [D]) if $M \leftleftarrows F_{1}^{\phi} \oplus F_{2}^{q}$ for $p+q=n+1$. This is equivalent to saying that $\left(F_{1} * F_{2}\right)^{n}=M$ and $\left(F_{1} * F_{2}\right)^{n+1}=0$. The following is easy to prove (See $[\mathrm{S}-\mathrm{Z}])$.

1.5. Lemma 1.5.1. Let $F_{1}, F_{2}$ be two filtrations of an object $M$.

(i) $([\mathrm{S}-\mathrm{Z}])\left(F_{1} * F_{2}\right)^{p}\left(g r_{F_{1}}^{k}\right)=F_{2}^{p-k}\left(g r_{F_{1}}^{k}\right)$

(ii) Let $G$ be another filtration of $M$. Then $G=F_{1} * F_{2}$ if and only if $F_{1}\left(g r_{G}^{k}\right)$ and $F_{2}\left(g r_{G}^{k}\right)$ is k-opposed for any $k$. 
1.6. As a generalization of the amalgum of two filtrations, we make the following definition.

Definition 1.6.1. For a family of filtrations $F_{1}, \ldots, F_{n}$ of an object $M$, we define

$$
\begin{aligned}
& I\left(F_{1}, \ldots, F_{n}\right)^{k}=\sum_{k=\Sigma p_{j}}(\overbrace{j=1}^{n} F_{j}^{p_{j}}) \\
& S\left(F_{1}, \ldots, F_{n}\right)^{k}=\bigcap_{k+n-1=\Sigma p_{j}}\left(\sum_{j=1}^{n} F_{j}^{b_{j}}\right) .
\end{aligned}
$$

Remark 1.6.2. $I$ and $S$ are the dual notions to each other, i. e. for an exact contravariant functor $T$, we have

$$
T\left(I\left(F_{1}, \ldots, F_{n}\right)\right)=S\left(T\left(F_{1}\right), \ldots, T\left(F_{n}\right)\right)
$$

$$
T\left(S\left(F_{1}, \ldots, F_{n}\right)\right)=I\left(T\left(F_{1}\right), \ldots, T\left(F_{n}\right)\right) .
$$

Since the proof of the following lemma is simple and similar to that of Lemma 1.3.1, we omit its proof.

Lemma 1.6.2. Let $F_{1}, \ldots, F_{n}$ be a family of filtrations. Then we have

(i) $\quad I\left(F_{1}, \ldots, F_{n}\right) \subset S\left(F_{1}, \ldots, F_{n}\right)$.

(ii) $I\left(F_{1}, \ldots, F_{n}\right) \subset I\left(I\left(F_{1}, \ldots, F_{l}\right), F_{l+1}, \ldots, F_{n}\right)$

(iii) $S\left(F_{1}, \ldots, F_{n}\right) \supset S\left(S\left(F_{1}, \ldots, F_{l}\right), F_{l+1}, \ldots, F_{n}\right)$.

Definition 1.6.3. A family $\left\{F_{1}, \ldots, F_{n}\right\}$ of filtrations is called distributive if $I\left(F_{1}, \ldots, F_{n}\right)=S\left(F_{1}, \ldots, F_{n}\right)$.

The naming comes from Remark 1.7.3. A single filtration and a couple of filtrations are distributive (Lemma 1.3.1).

1.7. We shall study the property of distributive families of filtrations. The following is a key lemma.

Proposition 1.7.1. A family $\left\{F_{1}, \ldots, F_{n}\right\}$ of filtrations of $M$ is distributive if and only if the following two conditions are satisfied.

(i) For any $q, \quad\left\{F_{1} \cap F_{n}^{q}, \ldots, F_{n-1} \cap F_{n}^{q}\right\}$ is a distributive family of filtrations of $F_{n}^{q}$ and $I\left(F_{1} \cap F_{n}^{q}, \ldots, F_{n-1} \cap F_{n}^{q}\right)=I\left(F_{1}, \ldots, F_{n-1}\right) \cap F_{n}^{q}$. 
(ii) For any $q,\left\{\left(F_{1}+F_{n}^{q}\right) / F_{n}^{q}, \ldots,\left(F_{n-1}+F_{n}^{q}\right) / F_{n}^{q}\right\}$ is a distributive family of filtrations of $M / F_{n}^{q}$ and we have

$$
I\left(\left(F_{1}+F_{n}^{q}\right) / F_{n}^{q}, \ldots,\left(F_{n-1}+F_{n}^{q}\right) / F_{n}^{q}\right)=\left(I\left(F_{1}, \ldots, F_{n-1}\right)+F_{n}^{q}\right) / F_{n}^{q} .
$$

Proof. Since the implication (i) + (ii) $\Rightarrow$ " $\left\{F_{1}, \ldots, F_{n}\right\}$ is distributive" is easily proven, we shall show only the converse implication. Since (i) and (ii) are the dual statements, we shall show only (i). Let $\mathscr{F}$ be the family $\left\{F_{1}, \ldots, F_{n-1}\right\}$. Then we have

$$
\begin{aligned}
& I\left(\mathscr{F} \cap F_{n}^{q}\right) \subset I(\mathscr{F}) \cap F_{n}^{q} \\
& S\left(\mathscr{F} \cap F_{n}^{q}\right) \subset S(\mathscr{F}) \cap F_{n}^{q}
\end{aligned}
$$

Hence it is enough to show

$$
\left(A_{p, q}\right): S(\mathscr{F})^{p} \cap F_{n}^{q} \subset I\left(\mathscr{F} \cap F_{n}^{q}\right)^{p}
$$

This is clear for $p \gg 0$ or $q \gg 0$. Therefore it is enough to prove $\left(A_{p, q+1}\right)+\left(A_{p+1, q}\right) \Rightarrow\left(A_{p, q}\right)$. Now, we have, since $\left\{\mathscr{F}, F_{n}\right\}$ is distributive

$$
\begin{aligned}
S(\mathscr{F})^{p} \cap F_{n}^{q} \subset\left(S(\mathscr{F}) * F_{n}\right)^{p+q} \subset S\left(\mathscr{F}, F_{n}\right)^{p+q} \\
=I\left(\mathscr{F}, F_{n}\right)^{p+q} \\
\quad=\sum_{\sum_{j=1}^{n-1} p_{j}+k=p+q}\left(\bigcap_{j=1}^{n-1} F_{j}^{p_{j}} \cap F_{n}^{k}\right)
\end{aligned}
$$

By dividing the summation into three parts

$$
\left\{\sum p_{j}=p, k=q\right\},\left\{\sum p_{j} \leq p-1, k \geq q+1\right\}
$$

and

$$
\left\{\sum p_{j} \geq p+1, k \leq q-1\right\}
$$

we obtain

$$
S(\mathscr{F})^{p} \cap F_{n}^{q} \subset I\left(\mathscr{F} \cap F_{n}^{q}\right)^{p}+F_{n}^{q+1}+I(\mathscr{F})^{p+1} .
$$

This implies

$$
S(\mathscr{F})^{p} \cap F_{n}^{q} \subset I\left(\mathscr{F} \cap F_{n}^{q}\right)^{p}+I(\mathscr{F})^{p} \cap F_{n}^{q+1}+I(\mathscr{F})^{p+1} \cap F_{n}^{q}
$$

Then $\left(A_{p, q+1}\right)$ and $\left(A_{p+1, q}\right)$ imply that the last two terms are contained in $I\left(\mathscr{F} \cap F_{n}^{q}\right)^{p}$, and hence we obtain $\left(A_{p, q}\right)$.

Q.E.D.

Proposition 1.7.2. Let $\left\{F_{1}, \ldots, F_{n}\right\}$ be a distributive family of filtrations. Then we have

(i) $\left\{F_{1}, F_{1}, \ldots, F_{n}\right\}$ is also distributive. 
(ii) $\left\{F_{1} * F_{2}, F_{3}, \ldots, F_{n}\right\},\left\{F_{1}+F_{2}, F_{3}, \ldots, F_{n}\right\}$ and $\left\{F_{1} \cap F_{2}, F_{3}, \ldots, F_{n}\right\}$ are also distributive. Here $F_{1}+F_{2}$ (resp. $F_{1} \cap F_{2}$ ) is the filtration defined by $\left(F_{1}+F_{2}\right)^{p}=F_{1}^{p}+F_{2}^{p} \quad\left(\operatorname{resp} .\left(F_{1} \cap F_{2}\right)^{p}=F_{1}^{p} \cap F_{2}^{p}\right)$.

(iii) For $q$ and $j$ we denote by $\tilde{F}_{j}^{q}$ the filtration given by $\left(\tilde{F}_{j}^{q}\right)^{k}=M, F_{j}^{q}, 0$ for $k=0,1,2$. Then $\left\{F_{1}, \ldots, F_{n}, \tilde{F}_{j}^{q}\right\}$ is distributive.

(iv) we have

$$
\left(F_{i}^{p}+F_{j}^{q}\right) \cap F_{k}^{r}=\left(F_{i}^{p} \cap F_{k}^{r}\right)+\left(F_{j}^{q} \cap F_{k}^{r}\right) .
$$

Since the proof is more or less direct, we omit it.

Remark 1. 7. 3. $\left\{F_{1}, \ldots, F_{n}\right\}$ is a distributive family if and only if the lattice (by + and $\cap$ ) generated by $F_{j}^{q}$ s is distributive (i. e. $(a+b) \cap$ $c=(a+\mathrm{c}) \cap(\mathrm{b}+\mathrm{c}))$.

1.8. Lemma 1.8.1. Let $\left\{F_{1}, \ldots, F_{n}\right\}$ be a family of filtrations of M. We assume $I\left(F_{1}, \ldots, F_{n}\right)^{l}=M$ and $S\left(F_{1}, \ldots, F_{n}\right)^{l+1}=0$. Then we have

(i) Setting, for $p_{1}, \ldots, p_{n}$ with $l=\sum p_{j}, H^{p_{1}, \ldots, p_{n}}=\bigcap_{j=1}^{n} F_{j}^{p_{j}}$, we have

$$
M \approx{ }_{l=p_{1}+\cdots+p_{n}} H^{p_{1} \cdots \cdots p_{n}}
$$

(ii) $\quad F_{k}^{q} \leftleftarrows \bigoplus_{q \leqq p_{k}} H^{p_{1}, \ldots, p_{n}}$

where the indices run over $l=\sum p_{j}$ and $p_{k} \geqq q$.

Proof. Set $\tilde{H}^{p_{1} \ldots, p_{n}}=M / \sum_{j=1}^{n} F_{i}^{p_{i}+1}$. Then the image of $\varphi: \oplus H^{p} \rightarrow M$ is $I\left(F_{1}, \ldots, F_{n}\right)^{l}$ and the kernel of $\phi: M \rightarrow \oplus \tilde{H}^{b}$ is $S\left(F_{1}, \ldots, F_{n}\right)^{l+1}$. Hence we have $\oplus H^{p} \rightarrow M \gg \oplus \tilde{H}^{p}$. On the other hand, $H^{p} \rightarrow \tilde{H}^{p^{\prime}}$ is zero for $p \neq p^{\prime}$. Therefore $H^{p} \rightarrow \tilde{H}^{p}$ is an isomorphism and we have $\oplus H^{p} \rightarrow M$ $\Im \oplus \tilde{H}^{p}$. Now we have $\phi\left(F_{j}^{q}\right) \subset \bigoplus_{p_{j} \geq q} \tilde{H}^{p}$ and hence $F_{j}^{q} \subset \sum_{p_{j} \geq q} H^{p}$ 。 The $\begin{array}{ll}\text { other inclusion is evident and we have (ii). } & \text { Q. E. D. }\end{array}$

Definition 1.8.2. If the assumption in Lemma 1.8 .1 is verified we say $\left\{F_{1}, \ldots, F_{n}\right\}$ is l-opposed.

Proposition 1.8.3. Let $\left\{F_{1}, \ldots, F_{n}\right\}$ be a distributive family of filtrations of $M$. Set $G=I\left(F_{1}, \ldots, F_{n}\right)=S\left(F_{1}, \ldots, F_{n}\right)$. Then we have 
(i) $\left\{F_{j}\left(g r_{G}^{k}\right)\right\}_{j=1, \ldots, n}$ is k-opposed.

(ii) Setting $H^{p_{1}, \ldots, p_{n}}=\bigcap_{j} F_{j}^{p_{j}}\left(g g_{G}^{k}\right)$ with $k=\sum p_{j}$, we have $\cap F_{j}^{p_{j}} \rightarrow H^{p_{1} \ldots \ldots, p_{n}}$.

Corollary 1.8.3. Let $\left\{F_{1}, \ldots, F_{n}\right\}$ be a distributive family of filtrations of a semi-simple object $M$. Then there is a direct sum decomposition

$$
M=\oplus I^{p_{1} \ldots \ldots p_{n}}
$$

such that

$$
F_{j}^{q}=\bigoplus_{p_{j} \geq q} I^{p_{1} \ldots \ldots p_{n}}
$$

Proof. We take $I^{p_{1}, \ldots, p_{n}}$ such that $I^{p_{1} \ldots, p_{n}} \subset \cap F_{j}^{p_{j}}$ and $I^{p_{1} \ldots, p_{n}} \rightarrow$ $H^{p_{1}, \ldots, p_{n}}$ is an isomorphism. Then the last proposition implies the desired result.

Definition 1.8.4. We call $\left\{I^{p_{1} \ldots, p_{n}}\right\}$ the splitting of $\left\{F_{1}, \ldots, F_{n}\right\}$.

1. 9. Now let $M$ be a finite-dimensional vector space over $\boldsymbol{R}$, and $\left\{W^{1}, \ldots, W^{n}\right\}$ a distributive family of increasing filtrations of $M$. For a multi-index $\alpha=\left(\alpha_{1}, \ldots, \alpha_{n}\right)$, we set $W_{\alpha}=\bigcap_{j} W_{\alpha_{j}}^{j}$. Let $T$ be a set and $f_{\alpha}(t)$ a non-negative valued function on $T$. We assume

(1.9.1) For $\alpha$ and $\beta$ such that $\beta \leqq \alpha$ (i. e. $\beta_{j} \leqq \alpha_{j}$ for any $j$ ), there exists a positive constant $C$ such that $f_{\beta}(t) \leqq C f_{\alpha}(t)$.

Let ||$_{t}$ be a family of norms parametrized by $t \in T$. We fix a norm | | on $M$.

Lemma 1.9.1. Let $M=\oplus I_{\alpha}$ be a decomposition such that $W_{\alpha}=\underset{\beta \leq \alpha}{\oplus} I_{\beta}$. We assume

(1.9.2) For $u \in I_{\alpha}$, there exists a constant $C$ such that $|u|_{t} \leqq C f_{\alpha}(t)$ for any $t \in T$.

Then we have the following.

(1.9.3) There exists a constant $C$ such that for any $u \in M$, if we write $u=\sum u_{\alpha}$ with $u_{\alpha} \in I_{\alpha}$, then we have

$$
|u|_{t} \leqq C \sum_{\alpha} f_{\alpha}(t)\left|u_{\alpha}\right| \text { for any } t \text {. }
$$


Proof. Let us take a base $\left\{u_{j}\right\}$ of $I_{\alpha^{*}}$ Then there exists $C>0$ such that $\left|u_{j}\right|_{t} \leqq C f_{\alpha}(t)$. Hence, for any $u=\sum \lambda_{j} u_{j}$ in $I_{\alpha},|u|_{t} \leqq C \sum$ $\left|\lambda_{j}\right| f_{\alpha}(t) \leqq C^{\prime}|u| f_{\alpha}(t)$. Hence there exists a constant $C>0$ such that for any $\alpha$ and any $u \in I_{\alpha},|u|_{t} \leqq C f_{\alpha}(t)|u|$. Thus if $u=\sum u_{\alpha}$, we have $|u|_{t} \leqq \sum\left|u_{\alpha}\right|_{t} \leqq C \sum_{\alpha} f_{\alpha}(t)\left|u_{\alpha}\right|$.

Corollary 1.9.2. The following condition on ||$_{t}$ does not depend on the choice of a splitting $M=\oplus I_{\alpha}$ such that $W_{\alpha}=\bigoplus_{\beta \leqq \alpha} I_{\beta}$.

(1.9.5) there exists a constant $G>0$ such that for any $u=\sum u_{\alpha}$ with $u_{\alpha} \in I_{\alpha}$, we have

(1.9.5.1) $\quad|u|_{t} \leqq C \sum f_{\alpha}(t)\left|u_{\alpha}\right|$ for any $t \in T$.

(1.9.5.2) $\sum f_{\alpha}(t)\left|u_{\alpha}\right| \leqq C|u|_{t}$ for any $t \in T$.

Definition 1.9.3. If the condition (1.9.5) is satisfied, we write $|u|_{t} \sim f_{\alpha}(t)$ on $u \in W_{\alpha}$ and $t \in T$.

\section{§2. Polarized Hodge Structure}

2.1. Let $H_{R}$ be a finite-dimensional $\mathbb{R}$-vector space with a nondegenerate bilinear form $S(*, *)$ and let $H_{C}$ be the complexification $\boldsymbol{C} \otimes_{R} H_{R}$ of $H_{R}$. Let us fix a weight $n \in \mathbb{Z}$ and assume

$$
S(u, v)=(-)^{n} S(v, u) \text {. }
$$

Let $G=\mathrm{O}\left(S, H_{c}\right)$ and let $G_{R}=\mathrm{O}\left(S, H_{R}\right)$ be its real form. Let us denote by $\mathfrak{g}$ and $\mathfrak{g}_{R}$ the Lie algebras of $G$ and $G_{R}$, respectively.

Let $\check{D}$ be the classifying space of Hodge filtrations, that is,

$$
\begin{aligned}
& \check{D}=\left\{F ; F \text { is a finite filtration of } H_{C},\right. \\
& \left.F^{\not \perp}=F^{n+1-p} \text { and } \operatorname{dim} F^{p} \text { are the given one }\right\} .
\end{aligned}
$$

Here $\perp$ denotes the orthogonal complement with respect to $S$. Then $\check{D}$ is a projective homogeneous space of $G_{C}$. A Hodge filtration $F$ is called a Hodge structure if $F$ and its complex conjugate $\bar{F}$ is $n$ opposed. Then $H_{c}=\bigoplus_{n=p+q} H^{p, q}$ with $H^{p, q}=F^{p} \cap \bar{F}^{q}$. We define the Weil operator $\mathrm{C} \in G_{\boldsymbol{R}}$ by $\left.\mathrm{C}\right|_{H^{p, q}}=i^{p-q}$. We say that $S$ is a polarization of the Hodge structure $\left(H_{R}, F\right)$ if the Hermitian form $S(\mathrm{C} u, \bar{u})$ is positive definite. In this case, we define the norm $|*|_{F}$ by 


$$
|u|_{F}^{2}=S(\mathrm{C} u, \bar{u}) .
$$

We denote by $D$ the set of $F \in \check{D}$ such that $S$ gives a polarization of $F$. Then $D$ is a homogeneous space of $G_{R}$ and the isotropy subgroup is compact.

2.2. Let $N$ be a nilpotent element in $\mathfrak{g}_{R}$.

Then there exists a unique finite filtration $W$ of $H_{c}$ such that

$$
\begin{aligned}
& N W_{k} \subset W_{k-2} \\
& N^{k}: g r_{k}^{W} \rightarrow g r_{-k}^{W} \text { for } k \geqq 1 .
\end{aligned}
$$

This filtration $W$ is denoted by $W(N)$ and called the monodromy weight filtration of $N$.

We have easily

$$
\mathrm{W}(N)_{k}^{\perp}=W(N)_{-1-k} \text {. }
$$

For $k \geqq 0$, the kernel of $N^{k+1}: g r_{k}^{W} \rightarrow g r_{-k-2}^{W}$ is denoted by $P_{k}(N)$ and called the primitive part. Then we have

$$
g r_{k}^{W} \cong \bigoplus_{j \geqq 0} P_{|k|+2 j}(N) \text {. }
$$

2.3. Let $I$ be a mutually commuting set of nilpotent elements of $\mathfrak{g}_{R}$. We set

$$
\mathrm{G}(I)=\left\{\sum_{N \in I} t_{N} N ; t_{N}>0\right\}
$$

Definition 2.3.1. For an $F \in \check{D}$ and $I$ as above, we say that $\{I, F\}$ forms a nilpotent orbit if the following conditions are satisfied.

$$
N F^{p} \subset F^{p-1} \text { for any } N \in I \text {. }
$$

(2.3.3) There exists $N_{0} \in \mathrm{G}(I)$ such that $e^{i N} F \in D$ for $N \in N_{0}+\mathrm{G}(I)$.

The following theorem is due to W. Schmid.

Theorem 2.3.2 ([S]). Let $N$ be a nilpotent element in $\mathfrak{g}_{R}, F \in \check{D}$ and assume that $\{N, F\}$ forms a nilpotent orbit. Let $W$ be the monodromy weight filtration of $N$. Then we have the following properties.

(2.3.4) $(F, W)$ is a mixed Hodge structure of weight $n$, i.e. $\left(F\left(g r_{k}^{W}\right), \bar{F}\left(g r_{k}^{W}\right)\right)$ is $(n+k)$-opposed.

(2.3.5) The bilinear form $S\left(u, N^{k} v\right)$ on $g r_{k}^{W}$ gives a polarization of the Hodge structure on the primitive part $P_{k}(N)$. 
We have the partial converse of this theorem. Let $F \in \check{D}, N$ a nilpotent element of $\mathfrak{g}_{R}$ and let $W$ be the monodromy weight filtration of $N$ and $P_{k}$ the primitive part.

Lemma 2.3.3 ([C-K $])$. Assume the following conditions.

(2.3.6) $(F, W)$ is a mixed Hodge structure of weight $n$.

(2.3.7) $\quad(F, W)$ is $\boldsymbol{R}$-split, that is, if we set $I^{p, q}=F^{b} \cap \bar{F}^{q} \cap W_{p+q-n}$, then $F^{p}=\bigoplus_{q} I^{p, q}$ and $W_{k}=\bigoplus_{p+q \leqq k+n} I^{p, q}$.

(2.3.8) $S\left(u, N^{k} v\right)$ gives a polarization of the primitive part $P_{k}$. Then $e^{i t N} F \in D$ for $t>0$.

The following theorem is due to Gattani-Kaplan.

Theorem 2.3.4 ([C-K]). Let $I$ be a mutually commuting set of nilpotent elements of $\mathrm{g}_{R}, F \in \check{D}$ and assume that $\{I, F\}$ forms a nilpotent orbit. Then we have

(2.3.9) For any $J \subset I$, there exists a filtration $W(J)$ such that $W(J)$ is the monodromy filtration of any $N \in \mathrm{C}(J)$.

(2.3.10) There exists $g \in G$ with the following properties

(2.3.10.1) $g$ commutes with $I$.

(2.3.10.2) $\left.\quad g\right|_{g r k} ^{W(I)}=\mathrm{id}$ for any $k$.

(2.3.10.3) If we set $F_{0}=g F$, then $\left\{F_{0}, W(I)\right\}$ is $\mathbb{R}$-split and $e^{i N} F_{0} \in D$ for any $N \in \mathrm{C}(I)$.

2.4. Admitting these results in $\S 2.3$, we shall start our arguments by the following lemma.

Lemma 2.4.1. Let $F \in \check{D}$ and let $I$ be a commuting finite set of nilpotent elements in $\mathfrak{g}_{\boldsymbol{R}}$. Assume that $\{I, F\}$ forms a nilpotent orbit. Then, for any decreasing sequence $I=I_{0} \supset I_{1} \supset$. . $\supset I_{m}$ of subsets $I,\left\{F, W\left(I_{0}\right)\right.$, $\left.W\left(I_{1}\right), \ldots, W\left(I_{m}\right)\right\}$ is a distributive family.

Proof. We shall prove this by the induction on $m$. Therefore, we may assume that

(2.4.1) $\left\{W\left(I_{j}\right)\right\}_{j \geqq 1}$ is a distributive family. 
By (2.3.10), there exists $g \in G$ commuting with $I$ and satisfying the conditions $(2.3 .10 .2) \sim(2.3 .10 .3)$. Since $g W\left(I_{k}\right)=W\left(I_{k}\right)$, we may replace $F$ with $g F$, which is $\boldsymbol{R}$-split. Hence we may assume from the beginning that $(F, W(I))$ is $\boldsymbol{R}$-split. Set $I^{p, q}=F^{b} \cap \bar{F}^{q} \cap$ $W_{p+q-n}(I)$. Then we have $H_{c}=\oplus I^{p, q}$ and $F^{p}=\bigoplus_{q} I^{p, q}$.

Let us define $Y_{1}, Y_{2} \in \operatorname{End}\left(H_{C}\right)$ by

$$
\left.Y_{1}\right|_{I^{p, q}}=p \text { and }\left.Y_{2}\right|_{I^{p, q}}=q \text {. }
$$

Then since $N I^{p, q} \subset I^{p-1, q-1}$ for $N \in I$, we have $\left[Y_{1}, N\right]=-\mathrm{N}$, $\left[Y_{2}, N\right]=-N$ for $N \in I$. Hence $Y_{1}$ and $Y_{2}$ preserves the filtrations $F$ and $W\left(I_{k}\right)$. By setting $I_{k}^{p, q}=W\left(I_{k}\right) \cap I^{p, q}$, we have $W\left(I_{k}\right)=\bigoplus_{p, q} I_{k}^{p, q}$.

Now by the assumption $\left\{W\left(I_{j}\right)\right\}_{j \geqq 1}$ is a distributive family, and hence $\left\{I_{j}^{p, q}\right\}_{j \geqq 1}$ is a distributive family of filtrations on $I^{p, q}$. This immediately implies the desired result.

Q. E. D.

Theorem 2.4.2. Let $K$ be a compact subset of $\check{D}$ and let $I=\left\{N_{1}, \ldots\right.$, $\left.N_{l}\right\}$ be a commuting set of nilpotent elements in $g_{R}$. We set $I_{j}=\left\{N_{i}\right.$; $i \geqq j\}$.

We assume the following conditions.

(2.4.3.1) If $F \in K$ and $N \in I$ then $N F^{p} \subset F^{p-1}$

(2.4.3.2) For $F \in K$ and $N \in \mathrm{C}(I)$ we have $e^{i N} F \in D$

(2.4.3.3) For any $p, k$ and $J \subset I, \operatorname{dim} F^{b} \cap W_{k}(J)$ does not depend on $F \in K$.

Then for any $\varepsilon>0$ we have

$$
\begin{aligned}
& |u|_{e^{i N} F}^{2} \sim t_{1}^{p_{1}}\left(t_{2} / t_{1}\right)^{p_{2}} \ldots\left(t_{l} / t_{l-1}\right)^{p_{l}} \\
& \text { on } u \in \overbrace{j=1}^{l} W_{p_{j}}\left(I_{j}\right) \text { and } N=\sum_{j=1}^{l} t_{j} N_{j} \text { with } t_{1}>\varepsilon, \\
& t_{j} / t_{j-1}>\varepsilon \text { for } 2 \leqq i \leqq l .
\end{aligned}
$$

For this notation, see Definition 1.9.3.

Corollary 2.4.3. Under the same notation, we have

$$
\left|e^{i N} u\right|_{e^{i N} F}^{2} \sim t_{1}^{p_{1}}\left(t_{2} / t_{1}\right)^{p_{2}} \ldots\left(t_{l} / t_{l-1}\right)^{p_{l}}
$$

on $u \in \bigcap_{j=1}^{l} W_{p_{j}}\left(I_{j}\right)$ and $N=\sum_{j=1}^{l} t_{j} N_{j}$ with $t_{1}>\varepsilon, t_{j} / t_{j-1}>\varepsilon$ for $2 \leqq j \leqq l$. 
Proof. We shall prove this by the induction on $l$. Set $W=W(I)$. Let us take a semi-simple element $Y$ in $g_{R}$ such that

$$
\begin{aligned}
& {[Y, N]=-2 N \text { for } N \in I} \\
& \left.Y\right|_{g_{k}^{W}}=k \cdot \text { id }
\end{aligned}
$$

For example, take $Y_{1}+Y_{2}-n$ in the proof of preceding lemma. If $V_{k}$ denotes the eigen-space of $Y$ with eigenvalue $k$, then $W_{k}=\bigoplus_{j \leq k} V_{j}$. Now, (2.4.5) implies

$$
\begin{aligned}
& e^{i s N}=s^{-Y / 2} e^{i N} S^{Y / 2} \\
& \text { for } N \in \mathrm{C}(I) \text { and } s>0 .
\end{aligned}
$$

Now, let $N=\sum_{j=1}^{l} t_{j} N_{j}$ with $t_{j}>\varepsilon$ be an element of $\mathrm{C}(I)$. We set $N^{\prime}=\sum_{j \geqq 2} t_{j} N_{j} / t_{1}$. Then we have $N=t_{1}\left(N_{1}+N^{\prime}\right)$. Therefore (2.4.7) implies

$$
\begin{aligned}
e^{i N} F & =t_{1}^{-Y / 2} e^{i\left(N_{1}+N^{\prime}\right)} t_{1}^{Y / 2} F \\
& =t_{1}^{-Y / 2} e^{i N^{\prime}}\left(e^{i N_{1}} t_{1}^{Y / 2} F\right) .
\end{aligned}
$$

Lemma 2.4.4. For any $\varepsilon>0$, let $K^{\prime}$ be the closure of $\left\{e^{i N_{1} S^{Y / 2}} F ; F \in K\right.$, $s>\varepsilon\}$. Then $(2.4 .3 .1) \sim(2.4 .3 .3)$ for $\left(K^{\prime}, I_{2}\right)$ are satisfied.

Admitting this lemma for a while, we shall continue the proof of the theorem. By the hypothesis of the induction, we can apply the theorem for $I_{2}$ and $K^{\prime}$. Therefore we have for $N^{\prime}=\sum_{j=2}^{l}\left(t_{j} / t_{1}\right) N_{j}$ with $t_{j} / t_{j-1}>\varepsilon(2 \leqq j \leqq l)$.

$$
\begin{aligned}
& |u|_{e^{i N^{\prime} F^{\prime}}}^{{ }^{\prime}} \sim\left(t_{2} / t_{1}\right)^{p_{2}} \ldots\left(t_{l} / t_{l-1}\right)^{p_{l}} \\
& \text { on } u \in \overbrace{j=2}^{l} W_{p_{j}}\left(I_{j}\right) \text { and } F^{\prime} \in K^{\prime} \text {. }
\end{aligned}
$$

If $F^{\prime}=e^{i N_{1}} t_{1}^{Y / 2} F$ with $F \in K, t_{1}>\varepsilon$, then (2.4.8) implies $e^{i N} F=t_{1}^{-Y / 2} e^{i N^{\prime}} F^{\prime}$ 。 Hence, $t_{1}^{Y / 2} \in G_{R}$ gives

$$
|u|_{e^{i N_{F}}}=\left|t_{1}^{Y / 2} u\right|_{e^{i N^{\prime} F^{\prime}}}
$$

Now, $W\left(I_{j}\right)$ is invariant by $Y$. Hence we have $W\left(I_{j}\right)=\bigoplus_{k}\left(W\left(I_{j}\right) \cap V_{k}\right)$. Since $\left\{W\left(I_{j}\right)\right\}_{2 \leqq j}$ is distributive, $\left\{W\left(I_{j}\right) \cap V_{k}\right\}_{j \geq 2}$ is also a distributive family of filtrations of $V_{k}$. Hence we can write

$$
V_{k}=\bigoplus_{p} U_{k, p}
$$


where $p=\left(p_{2}, \ldots, p_{l}\right) \in Z^{l-1}$ and

$$
W_{q}\left(I_{j}\right)=\bigoplus_{p_{j} \leq q} U_{k, p} \quad \text { for } \mathrm{j} \geqq 2 .
$$

Then (2.4.9) means that, fixing a norm | | of $H_{C}$,

$$
\begin{aligned}
& |u|_{e^{i N_{F}^{\prime}}}^{2} \sim \sum_{p, k}\left(t_{2} / t_{1}\right)^{p_{2}} \ldots\left(t_{l} / t_{l-1}\right)^{p_{l}}\left|u_{k, p}\right|^{2} \\
& \text { where } u=\sum u_{k, p} \text { with } u_{k, p} \in U_{k, p} .
\end{aligned}
$$

Since $t_{1}^{Y / 2} u=\sum_{k} t_{1}^{k / 2} u_{k, p}$, (2.4.10) implies

$$
|u|_{e^{i N_{F}}}^{2} \sim \sum\left(t_{2} / t_{1}\right)^{p_{2}} \ldots\left(t_{l} / t_{l-1}\right)^{p_{l}}\left|t_{1}^{k / 2} u_{k, p}\right|^{2} .
$$

This is nothing but the meaning of (2.4.4).

Q. E. D.

2.5. Proof of Lemma 2.4.4. We shall prove first

Sublemma 2.5.1. (i) $t^{-Y} F(t>0, F \in K)$ can be continued to a continuous function from $\{(t, F) ; t \geqq 0, F \in K\}$ into $\check{D}$.

(ii) If we set $F_{0}=\left.t^{-Y} F\right|_{t=0}$, then $F_{0}\left(g r^{W}\right)=F\left(g r^{W}\right)$ and $\left(F_{0}, W\right)$ is $\boldsymbol{R}$ split.

(iii) $e^{i N} F_{0} \in D$ for any $N \in \mathrm{G}(I)$.

Proof. Since $\operatorname{dim}\left(F^{p} \cap W_{k}\right)$ is a constant function in $F \in K, F^{b}\left(g r_{k}^{W}\right)$ forms a vector sub-bundle of $g r_{k}^{W}$ on $K$. Let $F_{k}^{b} \subset V_{k}$ be the inverse image of $F^{p}\left(g r_{k}^{W}\right)$ by the isomorphism $V_{k} \Im g r_{k}^{W}$. Then $F_{k}^{p}$ depends continuously on $F$. Hence, locally in $F$, there exists an isomorphism depending continuously on $F$

$$
\varphi^{p}: \bigoplus_{k} F_{k}^{p} \Im F^{p}
$$

such that

$$
\varphi^{p}\left(F_{k}^{p}\right) \subset F^{p} \cap W_{k}
$$

and

$$
\phi_{k}^{p}(u)=u-\varphi^{p}(u) \in W_{k-1} \text { for } u \in F_{k}^{p}
$$

Therefore

$$
\begin{aligned}
t^{-Y} F^{p} & =\bigoplus_{k}\left\{t^{-Y} \varphi^{p}(u) ; u \in F_{k}^{p}\right\} \\
& =\bigoplus_{k}\left\{t^{k-Y}\left(u+\phi_{k}^{p}(u)\right) ; u \in F_{k}^{p}\right\} .
\end{aligned}
$$

Since $\psi_{k}^{p}(u) \in W_{k-1}, t^{k-1-Y} \psi_{k}^{p}(u)$ is a polynomial in $t$, and hence $t^{k-Y}$. 
$\left(u+\psi_{k}^{p}(u)\right)$ is a polynomial in $t$, whose value at $t=0$ is $u$. Therefore we have (i) and $F_{0}^{b}=\bigoplus_{k}^{b} F_{k}^{b}$ Since $\left(F\left(g r_{k}^{W}\right), \bar{F}\left(g r_{k}^{W}\right)\right)$ is a Hodge structure of weight $n+k$, we have $V_{k}=\underset{p+q=k+n}{\bigoplus} F_{k}^{p} \cap \bar{F}_{k}^{q}$. Therefore $\left(F_{0}, W\right)$ is $\boldsymbol{R}$-split. Then (iii) is an immediate consequence of Theorem 2.3.2 and Lemma 2.3.3.

Q. E. D.

Now we resume the proof of Lemma 2.4.4. The condition (2.4.3.1) for $\left(K^{\prime}, I_{2}\right)$ is evident. We shall prove (2.4.3.2) and (2.4.3.3) for $\left(K^{\prime}, I_{2}\right)$.

If $F^{\prime} \in K^{\prime}$, then there are two cases by the preceding sublemma.

$$
\begin{aligned}
& F^{\prime}=e^{i N_{1}} t_{1}^{Y / 2} F \quad \text { for } F \in K, t_{1}>\varepsilon_{0} \\
& F^{\prime}=e^{i N_{1}} F_{0} \text { with } F_{0}=\left.s^{-Y} F\right|_{s=0} \text { for an } F \in K .
\end{aligned}
$$

In the first case, for $N^{\prime} \in \mathrm{C}\left(I_{2}\right), e^{i N^{\prime}} F^{\prime}=t_{1}^{Y / 2} e^{t_{1}\left(N_{1}+N^{\prime}\right)} F$ belongs to $D$ by the assumption (2.3.3.2) for $(K, I)$. In the second case $e^{i N^{\prime}} F^{\prime}=e^{i\left(N^{\prime}+N_{1}\right)}$ $F_{0}$ belongs to $D$ by Sublemma 2.5.1 (iii). For $J \subset I_{2}$, we shall calculate $\operatorname{dim} F^{\prime p} \cap W_{k}(J)$. In the first case, $\operatorname{dim} F^{\prime p} \cap W_{k}(J)=\operatorname{dim} F^{p} \cap W_{k}(J)$. In the second case, we shall show $\operatorname{dim}\left(F^{\prime p} \cap W_{k}(J)\right)=\operatorname{dim} F^{p} \cap W_{k}(J)$, which completes the proof of Lemma 2.4.4. Now, we shall use the fact that $\{F, W(J), W\}$ and $\left\{F_{0}, W(J), W\right\}$ are distributive (Lemma 2.4.1). We have

$$
\begin{aligned}
\operatorname{dim}\left(F^{\prime p} \cap W_{k}(J)\right) & =\operatorname{dim}\left(F_{0}^{b} \cap W_{k}(J)\right) \\
& =\sum_{j} \operatorname{dim}\left(F_{0}^{b}\left(g r_{j}^{W}\right) \cap W_{k}(J)\left(g r_{j}^{W}\right)\right) \\
& =\sum_{j} \operatorname{dim}\left(F^{p}\left(g r_{j}^{W}\right) \cap W_{k}(J)\left(g r_{j}^{W}\right)\right) \\
& =\operatorname{dim}\left(F^{p} \cap W_{k}(J)\right) .
\end{aligned}
$$

\subsection{Proof of Corollary 2.4.3.}

For $u \in \overbrace{j=1}^{l} W_{p_{j}}\left(I_{j}\right)$, we have

$$
\begin{aligned}
\left|e^{i N} u\right|_{e^{i N F}}^{2} & \leqq \Sigma\left(\frac{1}{\alpha !} t_{1}^{\alpha_{1}} \ldots t_{l}^{\alpha_{l}}\left|N_{1}^{\alpha_{1}} \ldots N_{l}^{\alpha_{l}} u\right|_{e^{i N_{F}}}\right)^{2} \\
& \leqq \text { const. } t_{1}^{p_{1}}\left(t_{2} / t_{1}\right)^{p_{2}} \ldots\left(t_{l} / t_{l-1}\right)^{p_{l}},
\end{aligned}
$$

because $N_{i} W_{k}\left(I_{j}\right) \subset W_{k}\left(I_{j}\right)$ and $N_{i} W_{k}\left(I_{j}\right) \subset W_{k-2}\left(I_{j}\right)$ for $i \in I_{j}$. Then 
Lemma 1.9.1 implies that there exists $C>0$ such that $\left|e^{i N} u\right|_{e^{i N_{F}}} \leqq$ $C|u|_{e^{i N_{F}}}$ Similarly we have $\left|e^{-i N} u\right|_{e^{i N F}} \leqq C|u|_{e^{i N_{F}}}$ These two imply $\left|e^{i N} u\right|_{e^{i N_{F}}} \sim|u|_{e^{i N_{F}}}$.

\section{§3. Variations of Polarized Hodge Structure}

3.1. Let $X$ be a complex manifold. A variation of Hodge structure of weight $n$ on $X$ is a couple $\left(H_{Z}, F\right)$ of a locally constant $\boldsymbol{Z}_{X}$-module $H_{Z}$ of finite rank and a finite filtration $\left\{F^{k}\right\}_{k \in Z}$ of $\mathcal{O}_{X} \underset{Z}{\otimes} H_{Z}$ by vector subbundles, satisfying

(3.1.1) At each point $x \in X,\left(H_{Z, x}, F(x)\right)$ is a Hodge structure of weight $n$.

$v F^{\phi} \subset F^{p-1}$ for any holomorphic vector field $v$.

A variation of Hodge structure is called polarized when a bilinear homomorphism $S: H_{\boldsymbol{Z}} \otimes H_{\boldsymbol{Z}} \rightarrow \boldsymbol{Q}_{X}$ is given in such a way that

$\left(H_{Z, x}, F(x), S_{x}\right)$ is a polarized Hodge structure at any $x \in X$.

3.2. Now, let $X$ be a complex manifold and $Y$ a closed analytic subset. Let $\left(H_{Z}, F, S\right)$ be a variation of Hodge structure of weight $n$ on $X \backslash Y$. For $x \in X \backslash Y$, let us denote by $\mathrm{C}(x)$ the Weil operator of the Hodge structure $\left(H_{Z, x}, F(x), S_{x}\right)$. Then

$$
\begin{aligned}
& \langle u \mid v\rangle_{x}=S_{x}(\mathrm{C}(x) u, \bar{v}), \quad u, v \in H_{C, x} \\
& \|u\|_{x}=\left(\langle u \mid u\rangle_{x}\right)^{1 / 2}
\end{aligned}
$$

defines the Hermitian metric on the vector bundle $H_{C}$, which depends really analytically on $x \in X \backslash Y$.

We shall discuss the behavior $\|u\|_{x}$ when $x$ goes to $Y$.

3. 3. Before studying the asymptotic behavior of variation of Hodge structure, we shall discuss the canonical extension of integrable connections. Let $X$ be a complex manifold and $Y$ a closed analytic subset and $\mathscr{F}$ a $\mathscr{D}_{X \backslash Y^{-}}$module coherent over $\mathcal{O}_{X \backslash Y}$. Let $j: X \backslash Y \subset X$ be the open embedding. We shall define the coherent $\mathcal{O}_{X}$-submodule $E_{\bar{X} \backslash Y}^{ \pm}(\mathscr{F})$ of $j_{*}(F)$ as follows. 
3. 3.1 Assume first $Y$ to be a normally crossing hypersurface. Then $E_{X \backslash Y}^{+}(\mathscr{F})$ is the unique locally free $\mathcal{O}_{X}$-submodule of $j_{*} \mathcal{O}_{X}$ which has the following property.

(3.3.1.1) For any holomorphic vector field $v$ tangent to $Y$, we have

$$
v E_{\bar{X} \backslash Y}^{ \pm}(\mathscr{F}) \sqsubset E_{\bar{X} \backslash Y}^{ \pm}(\mathscr{F}) \text {. }
$$

(3.3.1.2) At any non singular point $y$ of $Y$ and any vector field $v$ tangent to $Y$ such that $v \mid I_{Y} / I_{Y}^{2}=\mathrm{id}$, any eigenvalue of $v: E_{\bar{X} \backslash Y}^{ \pm}(\mathscr{F}) / I_{Y} E_{\bar{X} \backslash Y}^{ \pm}(\mathscr{F})$ is contained in $\{\lambda ; 0 \leqq \pm \operatorname{Re} \lambda<1\}$.

(3.3.1.3) $\left.\quad E_{\bar{X} \backslash Y}^{ \pm}(\mathscr{F})\right|_{X \backslash Y}=\mathscr{F}$

Then, $\mathscr{F} \mapsto E_{\bar{X} \backslash Y}^{ \pm}(\mathscr{F})$ is an exact functor. Moreover we have

$$
\begin{aligned}
& E_{X \backslash Y}^{+}(\mathscr{F}) \subset E_{\bar{X} \backslash Y}^{-}(\mathscr{F}) \\
& E_{\bar{X} \backslash Y}^{ \pm}(\mathscr{F})=\left(E_{X \backslash Y}^{\mp}(\mathscr{F} \vee)\right)^{\vee} . \quad \text { Here }{ }^{\vee}=\mathscr{H}_{6 m} \mathscr{O}(*, \mathcal{O}) .
\end{aligned}
$$

3.3.2 In general, let $f: X^{\prime} \rightarrow X$ be a proper morphism such that $Y^{\prime}=f^{-1} Y$ is a normally crossing hypersurface and that $X^{\prime} \backslash Y^{\prime} \rightarrow X \backslash Y$ is an isomorphism. We define $E_{\bar{X} \backslash Y}^{ \pm}(\mathscr{F})$ as

$$
E_{\bar{X} \backslash Y}^{ \pm}(\mathscr{F})=f_{*}\left(E_{\overline{X^{\prime} \backslash Y^{\prime}}}^{ \pm}\left(f^{-1} \mathscr{F}\right)\right) \text {. }
$$

This does not depend on the choice of $f$.

3.4. Now, let $X$ be a complex manifold, $Y$ a closed analytic subset of $X$ and $\left(H_{Z}, F, S\right)$ a variation of polarized Hodge structure.

We assume

(3.4.1) $\quad Y$ is a normal crossing hypersurface.

Let $Y=\cup_{j \in I} Y_{j}$ be the decomposition to irreducible components. For a finite subset $\alpha$ of $J$ set $Y_{\alpha}=\underset{j \in \alpha}{\cap} Y_{j}$.

Let $M_{j}$ be the monodromy of $H_{Z}$ around $Y_{j}$. Hence $M_{j}$ defines the automorphism of $H_{Z}$ on a neighborhood of $Y_{j}$. Then by [S], $M_{j}$ is quasi-unipotent. Taking a positive integer $m_{j}$ such that $M_{j}^{m_{j}}$ is unipotent, set $N_{j}=\frac{1}{m_{j}} \log M_{j}^{m_{j}}$. For a finite subset $\alpha$ let $W(\alpha)$ be the monodromy weight filtration of $\sum_{j \in \alpha} N_{j}$.

Then there exists an open neighborhood $U_{\alpha}$ of $Y_{\alpha}$ such that $W(\alpha)$ is the filtration of $\left.H_{Q}\right|_{\sigma_{\alpha} \backslash Y}$ 
Let us take $x_{0} \in X$ and $\alpha=\left\{j ; x_{0} \in Y_{j}\right\}=\left\{j_{1}, \ldots, j_{l}\right\}$. Let $f_{j}$ be the defining function of $Y_{j}$, and $y_{\nu}=-\log \left|f_{j_{\nu}}\right|$. Set $\alpha_{\nu}=\left\{j_{\mu} ; \mu \geq \nu\right\}$.

Theorem 3.4.1. There exists $M>0$ such that for any relatively compact subanalytic set $U$ in $X \backslash Y$ and for any $\varepsilon>0$, we have, for a flat section $u$ on $\left.H_{c}\right|_{U}$,

$$
|u|_{x}^{2} \sim y_{1}^{p_{1}}\left(y_{2} / y_{1}\right)^{p_{2}} \ldots\left(y_{l} / y_{l-1}\right)^{p_{l}}
$$

on $u \in \stackrel{\curlywedge}{\nu=1}^{l} W_{p_{j}}\left(\alpha_{j}\right), \quad x \in U, y_{\nu}>M(v=1, \ldots, l)$ and $y_{\nu} / y_{\nu-1}>\varepsilon$ for $\nu=2$, $\ldots, l$.

Theorem 3. 4.2. $\left\{E^{+}\left(\mathcal{O} \otimes W\left(\alpha_{1}\right)\right), \ldots, E^{+}\left(\mathcal{O} \otimes W\left(\alpha_{l}\right)\right)\right\}$ is a distributive family of filtrations of $E^{+}\left(\mathcal{O} \otimes H_{c}\right)$. Let us take a splitting

$$
E^{+}\left(H_{c}\right)=\oplus U_{p_{1}, \ldots, p_{l}}
$$

with $E^{+}\left(\mathcal{O} \otimes W_{p}\left(\alpha_{\nu}\right)\right)=\underset{p_{\nu} \leq p}{\bigoplus} U_{p_{1} \ldots, p_{l}}$ and a C Cor Hermitian norm $|*|$ on $E^{+}\left(\mathcal{O}_{X} \otimes H_{C}\right)$. Then on a neighborhood of $x_{0}$ we have

$$
\begin{aligned}
& \|u\|_{x}^{2} \sim \sum y_{1}^{p_{1}}\left(y_{2} / y_{1}\right)^{p_{2}} \ldots\left(y_{l} / y_{l-1}\right)^{p_{l}}\left|u_{p_{1} \ldots p_{l}}\right|^{2} \\
& \text { on } x \in\left\{x ; y_{j} / y_{j-1}>\varepsilon \text { for } j=2, \ldots, l\right\},
\end{aligned}
$$

where $u=\sum u_{p_{1} \ldots p_{l}}$ is a section of the vector bundle $E^{+}(\mathcal{O} \otimes H)$ and

$$
u_{p_{1}, \ldots, p_{l}} \in U_{p_{1}, \ldots, p_{l}} \text {. }
$$

\section{§4. Proof of Theorem 3.4.1 and 3.4.2}

4.1. As the question is local, we may assume (4.1.1) $\quad X=\Delta^{l}$.

Here $\Delta$ is the unit disc $\{z \in \boldsymbol{C} ;|z|<1\}$.

$$
Y=\left\{z \in X ; z_{1} \ldots z_{l}=0\right\} .
$$

Considering a branched covering by $z_{j}^{1 / m_{j}}$ if necessary, we may assume from the beginning

(4.1.3) The monodromy $M_{j}$ is unipotent.

Let $p: \widetilde{X \backslash Y}=\boldsymbol{C}_{+}^{l} \rightarrow X \backslash Y$ be a universal covering given by $\left(\tau_{1}, \ldots, \tau_{l}\right) \mapsto$ 
$\left(z_{1}, \ldots, z_{l}\right)$ with $z_{j}=e^{2 \pi i \tau_{j}}$. We shall trivialize $p^{-1} H_{Z^{\circ}}$. Then at each point $\tilde{x} \in \widetilde{X \backslash Y}$, the Hodge filtration $F(p(\tilde{x}))$ on a fixed vector space $H_{c}$ is given so that this defines a holomorphic map

$\widetilde{\Phi}: \widetilde{X \backslash Y \rightarrow D}$

to the appropriate classification space $D$ of polarized Hodge structure (see $\S 2$ ). Let $\check{D} \supset D$ be the classification space of Hodge filtration. We use the notations $G, G_{R}$, and $\mathfrak{g}_{R}$ as in $\S 2$. Then $N_{j} \in \mathfrak{g}_{R}$ and $\widetilde{\Psi}(\tau)$ $=e^{-2 \pi i \Sigma \tau_{j}^{N}{ }_{j}} \widetilde{\Phi}(\tau)$ gives the holomorphic map $\widetilde{\Psi}: \widetilde{X \backslash Y} \rightarrow \check{D}$, which are invariant by $\tau \mapsto \tau+m\left(m \in \boldsymbol{Z}^{l}\right)$. Hence it decomposes

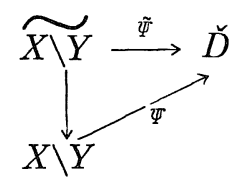

By the nilpotent orbit theorem ([S]), we have

(4. 1.6) $\Psi$ is continued to a holomorphic map $\Psi: X \rightarrow \check{D}$.

Set $F_{0}=\Psi(0)$ and define

$$
\widetilde{\Phi}_{0}(\tau)=e^{2 \pi i \Sigma \tau_{j} N_{j}} \circ F_{0} .
$$

Then Lemmas 8. 25 and 8. 27 in [S] say that there exists $g(\tau) \in G_{R}$ such that

$$
g(\tau) \widetilde{\Phi}(\tau)=a \text { is a fixed point of } D,
$$

and there exist $\beta, C, M>0$ such that

$$
\mathrm{d}_{M}\left(g(\tau) \widetilde{\Phi}_{0}(\tau), a\right) \leqq C\left(\sum \operatorname{Im} \tau_{j}\right)^{\beta}\left(\sum_{j} e^{-2 \pi \operatorname{Im} \tau_{j}}\right) \text { for } \operatorname{Im} \tau_{j}>M .
$$

Here $\mathrm{d}_{M}$ is a metric invariant by the action of a compact form $M$ of G.

In particular if $\delta$ is small enough we have

$$
\widetilde{\Phi}_{0}(\tau) \in D
$$

for $\tau$ with $\left(\sum \operatorname{Im} \tau_{j}\right)^{\beta}\left(\sum_{j} e^{-2 \pi \operatorname{Im} \tau_{j}}\right)<\delta$ and $\operatorname{Im} \tau_{j}>M$. Since $D$ is pseudo-convex to the horizontal direction (See [G-W], Lemma 4.2.1), $\left\{\tau \in \boldsymbol{C}_{+}^{m} ; \widetilde{\Phi}_{0}(\tau) \in D\right\}$ is also pseudo-convex. Moreover this contains

$$
E=\left\{\tau ;\left(\sum \operatorname{Im} \tau_{j}\right)^{\beta}\left(\sum_{j} e^{-2 \pi \operatorname{Im} \tau_{j}}\right)<\delta, \operatorname{Im} \tau_{j}>M\right\} .
$$

Remark that

(4.1.10) A connected tube domain which is pseudo-convex is convex. 
Since the convex hull of a connected component of $E$ contains $\left\{\tau ; \operatorname{Im} \tau_{j}>M^{\prime}\right\}$ for $M^{\prime} \gg 0$, we finally obtain

(4.1.11) $\widetilde{\Phi}_{0}(\tau) \in D$ for $\operatorname{Im} \tau_{j}>M^{\prime}$.

Proposition 4. 1. 1. $|u|_{\tilde{\Phi}_{0}(\tau)} \sim|u|_{\tilde{\Phi}_{(\tau)}}$ for $\operatorname{Im} \tau_{j}>M^{\prime}$.

If this proposition is proven, then Theorem 3.4.1 and Theorem 3.4.2 are immediate consequence of Theorem 2.4.2 and Corollary 2. 4.3 .

4. 2. In order to prove Proposition 4. 1. 1, we shall introduce $\delta\left(x, x^{\prime}\right)$, the function on $D \times D$.

For $x \in D$, let $\mathrm{G}(x)$ denotes the Weil operator of the Hodge structure at $x$. For $x, x^{\prime} \in D$ set

$$
\delta\left(x, x^{\prime}\right)=\operatorname{trG}(x)^{-1} \mathrm{G}\left(x^{\prime}\right)
$$

we have

$$
\left\langle\mathrm{G}(x)^{-1} \mathrm{G}\left(x^{\prime}\right) u \mid v\right\rangle_{x}=\langle u \mid v\rangle_{x^{\prime}}
$$

Hence for a suitable base, $\mathrm{G}(x)^{-1} \mathrm{G}\left(x^{\prime}\right)$ is a positive-definite symmetric matrix so that its eigenvalues are positive. Since $\mathrm{C}(x)^{-1} \mathrm{C}\left(x^{\prime}\right)$ is conjugate to its inverse, if $\lambda$ is an eigenvalue of $\mathrm{G}(x)^{-1} \mathrm{C}\left(x^{\prime}\right)$, then $\lambda^{-1}$ is also its eigenvalue with the same multiplicity. Hence we have

$$
\delta\left(x, x^{\prime}\right) \geq \operatorname{dim} H_{C}
$$

If the equality holds, then $\mathrm{C}(x)^{-1} \mathrm{C}\left(x^{\prime}\right)$ is unipotent. Hence we have

$$
\delta\left(x, x^{\prime}\right)=\operatorname{dim} H_{c} \text { implies } \mathrm{G}(x)=\mathrm{G}\left(x^{\prime}\right) \text {. }
$$

The relation (4.2.2) implies

$$
|u|_{x} \leqq \delta\left(x, x^{\prime}\right)|u|_{x^{\prime}}
$$

Lemma 4.2.1. (i) Let $Z$ be a complex manifold. Let $\varphi$ and $\varphi^{\prime}$ be horizontal holomorphic maps from $Z$ to $D$. Then $\delta\left(\varphi(z), \varphi^{\prime}(z)\right)$ is plurisubharmonic.

(ii) For any $G>0, x_{0} \in D,\left\{x \in D ; \delta\left(x, x_{0}\right) \leq C\right\}$ is a compact set.

Admitting this lemma for a while, we shall prove Proposition 4.1. 1. By (4.1.8), for $0<\varepsilon \ll \delta \ll 1$, we have

$$
\delta\left(g(\tau) \widetilde{\Phi}_{0}(\tau), a\right)<\operatorname{dim} H_{C}+\delta
$$

for $\tau \in E=\left\{\tau \in C_{+}^{l} ;\left(\sum \operatorname{Im} \tau_{j}\right)^{\beta}\left(\sum_{j} e^{-2 \pi \operatorname{Im} \tau}\right)<\varepsilon, \operatorname{Im} \tau_{j}>M\right\}$. This implies 


$$
\delta\left(\widetilde{\Phi}_{0}(\tau), \Phi(\tau)\right)<\operatorname{dim} H_{C}+\delta \text { for } \tau \in E .
$$

By Lemma 4.2.1 (i), $\left\{\tau ; \delta\left(\widetilde{\Phi}_{0}(\tau), \Phi(\tau)\right)<a\right\}$ is pseudo-convex. Hence (4. 1.10) implies

$$
\delta\left(\widetilde{\Phi}_{0}(\tau), \Phi(\tau)\right)<\operatorname{dim} H_{c}+\delta \text { for } \operatorname{Im} \tau_{j}>M^{\prime} .
$$

Now, it is enough to apply (4.2.5).

4. 3. Proof of Lemma 4.2. 1. (i) We may assume that $Z$ is an open set of $\boldsymbol{C}$. We fix a reference point $x_{0}$ of $X$. Set $\varphi(z)=g(z) x_{0}$ with a $C^{\infty}$-function $g(z) \in G_{R}$. Set $\mathrm{g}^{p_{1}-p}=\left\{A \in \mathrm{g} ; A F\left(x_{0}\right)^{k} \subset F\left(x_{0}\right)^{k+p}\right.$ and $A \overline{F\left(x_{0}\right)^{k}} \subset \overline{F\left(x_{0}\right)^{k-p}}$ \}. Then $\mathfrak{g}=\oplus \mathfrak{g}^{p,-p}$ and $\overline{\mathfrak{g}^{p,-p}}=\mathfrak{g}^{-p, p}$. That $\varphi$ is holomorphic is equivalent to

$$
g^{-1} g_{\bar{z}} \in \bigoplus_{p \geq 0} \mathrm{~g}^{p,-p}
$$

Here $g_{\bar{z}}$ is the derivative of $g$ with respect to $\bar{z}_{0}$ That $\varphi$ is horizontal is equivalent to

$$
g^{-1} g_{z} \in \bigoplus_{p \geq-1} \mathfrak{g}^{p_{,}-p}
$$

Hence $h=g^{-1} g_{z} \in \mathrm{g}^{0,0} \oplus \mathrm{g}^{-1,1}$. Set $h=h_{0}+h_{-1}$ with $h_{0} \in \mathrm{g}^{0,0}$ and $h_{-1} \in \mathrm{g}^{-1,1}$. Then $\bar{h}=g^{-1} g_{\bar{z}}=\bar{h}_{0}+h_{1}$ with $h_{1}=\bar{h}_{-1} \in \mathrm{g}^{1,-1}$. Then integrability condition implies

$$
h_{z}-\bar{h}_{\bar{z}}=[h, \bar{h}] \text {. }
$$

Hence we obtain

$$
\begin{aligned}
& h_{1 z}=\left[h_{1}, h_{0}\right] \\
& h_{-1 \bar{z}}=\left[\begin{array}{ll}
h_{-1}, & \bar{h}_{0}
\end{array} .\right.
\end{aligned}
$$

We have $\mathrm{C}=\mathrm{G}(\varphi(z))=g C_{0} g^{-1}$ where $C_{0}=\mathrm{G}\left(x_{0}\right)$. Hence

$$
\mathrm{C}_{z}=g\left[h, C_{0}\right] g^{-1} \text {. }
$$

Now, we have

$$
\left[h_{0}, C_{0}\right]=0
$$

$$
h_{1} C_{0}+C_{0} h_{1}=h_{-1} C_{0}+C_{0} h_{-1}=0 \text {. }
$$

Hence we obtain

$$
\mathrm{C}_{z}=2 g h_{-1} \mathrm{C}_{0} g^{-1} \text {. }
$$

The easy calculation shows
(4. 3.9)
$\mathrm{C}_{z \bar{z}}=2 g\left(h_{1} h_{-1}+h_{-1} h_{1}\right) C_{0} g^{-1}$ 。 
We define $\mathrm{C}^{\prime}=\mathrm{C}\left(\varphi^{\prime}(z)\right), g^{\prime}, h^{\prime}, h_{0}^{\prime}, h_{1}^{\prime}, h_{-1}^{\prime}$ similarly. Then $u=\delta(\varphi(z)$, $\left.\varphi^{\prime}(z)\right)=(-)^{n} \operatorname{trCC}^{\prime}$, and we have, setting $\varphi=g^{-1} g^{\prime}$,

$$
\begin{aligned}
u_{z \bar{z}}= & 2(-)^{n} \operatorname{tr}\left(\left(h_{1} h_{-1}+h_{-1} h_{1}\right) C_{0} \varphi C_{0} \varphi^{-1}\right. \\
& +\left(h_{1}^{\prime} h_{-1}^{\prime}+h_{-1}^{\prime} h_{1}^{\prime}\right) C_{0} \varphi^{-1} C_{0} \varphi+2 h_{-1} C_{0} \varphi h_{1}^{\prime} C_{0} \varphi^{-1} \\
& \left.+2 h_{1} C_{0} \varphi h_{-1}^{\prime} C_{0} \varphi^{-1}\right) .
\end{aligned}
$$

If we denote by $*$ the adjoint with respect to the Hermitian form $S\left(C_{0} u, \bar{v}\right)$, then we have $\operatorname{tr} A A^{*} \geq 0$ for $A \in \operatorname{End}\left(H_{C}\right)$. If we define ${ }^{t} A$ by $S\left({ }^{t} A u, v\right)=S(u, A v)$, then $A^{*}=C_{0}{ }^{\overline{ }} A C_{0}^{-1}$. Hence we obtain

$$
\operatorname{tr} A C_{0}^{\bar{t}} A C_{0}^{-1} \geq 0 \text {. }
$$

By setting

$$
A=\varphi^{-1} h_{ \pm 1}+h_{ \pm 1}^{\prime} \varphi^{-1},
$$

and using ${ }^{t} Y=-Y$ for $Y \in \mathrm{g}$, we obtain

$$
\begin{aligned}
\operatorname{tr} A C_{0}^{\bar{t}} A C_{0}^{-1}= & (-)^{n} \operatorname{tr}\left(h_{ \pm 1} h_{\mp 1} C_{0} \varphi C_{0} \varphi^{-1}+h_{\mp 1}^{\prime} h_{ \pm 1}^{\prime} C_{0} \varphi^{-1} C_{0} \varphi\right. \\
& \left.+h_{1} C_{0} \varphi h_{-1}^{\prime} C_{0} \varphi^{-1}+h_{-1} C_{0} \varphi h_{1}^{\prime} C_{0} \varphi^{-1}\right) \geq 0 .
\end{aligned}
$$

This shows $u_{z \bar{z}} \geq 0$.

4. 4. Proof of Lemma (ii). In order to prove this, let $\left\{x_{n}\right\}$ be a sequence in $D$ such that $\left\{\delta\left(x_{n}, x_{0}\right)\right\}_{n}$ is bounded and that $x_{n}$ converges to a point $x_{\infty} \in \check{D}$. It is enough to show $x_{\infty} \in D$. Then, $\langle\mid\rangle_{x_{n}}$ tends to a positive definite Hermitian form $\langle\mid\rangle_{\infty}$.

Let $H_{c}=\oplus H_{n}^{p, q}$ be the Hodge decomposition at $x_{n}$. We may assume $H_{n}^{p, q} \rightarrow H_{\infty}^{p, q}$. Since $H_{n}^{p, q}$ 's are orthogonal to each other with respect to $\langle\mid\rangle_{x_{n}},\left\{H_{\infty}^{p, q}\right\}$ is orthogonal to $\langle\mid\rangle_{\infty}$. Hence $\oplus H_{\infty}^{p, q} \rightarrow H_{C}$ is injective. Comparing the dimension, we have $H_{c}=\oplus H_{\infty}^{p, q}$. Therefore this is the Hodge decomposition at $x_{\infty}$ and $S\left(\mathrm{G}\left(x_{\infty}\right) u, \bar{v}\right)=\langle u \mid v\rangle_{\infty}$. Thus $x_{\infty}$ belongs to $D$.

\section{References}

[C-K] E. Cattani and A. Kaplan, Polarized mixed Hodge structures and the local monodromy of a variation of Hodge structure, Inv. Math., 67 (1982), 101-115.

[D] P. Deligne, Théorie de Hodge I, II and III, Actes, Congrès int. math., 1970, Tome 1, 425-430, Publ. Math. I.H.E. S., 40 (1972), 5-57 and ibid., 44 (1974), 5-77.

[G-S] P. Griffiths and W. Schmid, Locally homogeneous complex manifolds, Acta Math., 123 (1969), 253-302. 
[S] W. Schmid, Variation of Hodge structure: the singularities of the period mappings, Inv. Math., 22 (1973), 211-319.

[S-Z] J. Steenbrink and S. Zucker, Variation of mixed Hodge structure I, preprint.

[Z] S. Zucker, Hodge theory with degenerating coefficients: $L_{2}$ cohomology in the Poincaré metric, Annals of Math., 109 (1979), 415-476. 
